\title{
Effect of Dietary Supplement of Stearic Acid or Safflower Oil on the Plasma Lipids and Milk Fat in the Cow
}

\author{
Keiichi Tanaka, Iwao Nakajima and Hideo Hayashi \\ Faculty of Agriculture, Gifu University, Kagamigahara-shi, 504
}

(Received April 24, 1972)

\begin{abstract}
The effects of the isocaloric replacement of a part of the dietary concentrate mixture by stearic acid or safflower oil on rumen fermentation, blood plasma lipids and milk fat were investigated in feeding experiments using 4 lactating cows.

2) The safflower oil treatment resulted in an increase in the molar proportion of propionic acid and a decrease in that of acetic acid in the rumen liquor.

3) By the stearic acid treatment, the percentage of the milk fat tended to be increased, whereas the safflower oil treatment resulted in the decreased percentage of the milk fat.

4) By both treatments, the percentages of the fatty acids from 4:0 to 16:0 in the milk fat were decreased, while those of 18:0 and 18:1 in the milk fat were increased.

5) By the stearic acid treatment, the concentration of the plasma triglycerides from jugular vein increased, whereas the safflower oil treatment resulted in the decreased concentration of the plasma triglycerides from jugular vein. In both treatments, the concentrations of the plasma cholesterol esters, free cholesterol and phospholipids from jugular and subcutaneous abdominal veins were increased.

6) By both treatments, there were the increase in the percentage of 18:0 and the decrease in that of 16:0 of the plasma triglycerides and unesterified fatty acids. The safflower oil treatment resulted in increased percentage of 18:1 in the plasma triglycerides and unesterified fatty acids and also in an increased percentage of $18: 2$ and a decreased that of 18:3 in the plasma cholesterol esters. By the stearic acid treatment, the percentages of 18:0 and 18:1 in the plasma phospholipids tended to be increased and those of 16:0 and 18:2 did not decrease. By the safflower oil treatment, the percentage of $18: 2$ in the plasma phospholipids was increased.
\end{abstract}

In a previous study ${ }^{19}$, it was found that the type of dietary fat influenced the milk fat content and the fatty acid composition of the milk fat. A marked effect on the fatty acid composition in the milk fat was observed when the oil containing linoleic or linolenic acid was given as the dietary supplement. Also the increased dietary linoleic or linolenic acid resulted in the increased yield of oleic and stearic acids in milk, whereas the yield of linoleic and linolenic acids did not change. This is probably, in part, due to hydrogenation of these polyunsaturated fatty acids in the rumen prior to their absorption from the digestive tract.

In the present study, effects of feeding stearic acid or safflower oil on the pattern of rumen fermentation and on the plasina lipids were investigated in relation to milk fat secretion.

\section{Materials and Methods}

1) Experimental animals

Four Holstein cows (No. 1, 2, 3 and 4), which had calved 4 months before the beginning of the experiment, were used. Their body weights were as follows: cow No. 1: $787 \mathrm{~kg}$, cow 
Tanaka, Nakajima and Hayashi

Table 1. Mean daily intakes $(\mathrm{kg}$ ) of alfalfa hay cube and of each of the constituents of the concentrate mixtures

\begin{tabular}{l|c|c}
\hline \hline & $\begin{array}{c}\text { Unsupplemented } \\
\text { ration }\end{array}$ & $\begin{array}{c}\text { Ration } \\
\text { containing oil }\end{array}$ \\
\hline Alfalfa hay cube & $9.0(\mathrm{~kg})$ & $9.0(\mathrm{~kg})$ \\
Corn & 3.3 & 3.3 \\
Fish meal & 0.2 & 0.2 \\
Wheat bran & 4.2 & 2.6 \\
Extracted soybean meal & 1.0 & 1.0 \\
Minerals & 0.4 & 0.4 \\
Stearic acid or safflower oil & - & 0.4 \\
\hline
\end{tabular}

No. 2: $480 \mathrm{~kg}$, cow No. 3: $702 \mathrm{~kg}$, cow No. 4: $500 \mathrm{~kg}$. During the experiment, the cows were housed in a barn and yoked in stalls equipped with individual feeder. They were milked twice daily at 8:00 and 20:00. Their daily rations were given in 3 equal portions at 6:00, 11:00 and 16:00 each day. The oil was mixed with the rations at 6:00 and 16:00. Water was given ad libitum.

2) Experimental diets

The cows were given $9 \mathrm{~kg} /$ day of the alfalfa hay cube throughout the experiment. Three different concentrate mixtures were given to the cows. The basal concentrate mixture contained corn, fish meal, wheat bran, extracted soybean meal and minerals. The other two concentrate mixtures contained, in addition, stearic acid (obtained from Nakarai Chemicals, Ltd.) or safflower oil, and were compounded by isocalorically replacing part of the wheat bran with either stearic acid or safflower oil. The stearic acid was $89 \%$ pure and the safflower oil contained $79 \%$ linoleic acid. The daily intake of the various dietary components on the three feeding treatments are given in Table 1.

3) Experimental design

The experiment consisted of (1) the preliminary period, (2) the experimental period and (3) the post-experimental period. In the experimental period, cows No. 1 and No. 2 were fed on stearic acid and cows No. 3 and No. 4 on safflower oil. The duration of each treatment period was 21 days and the shifts between treatments were made abruptly.

4) Sampling and methods of analysis

Milk yields were recorded for the last five days of each treatment period. Composite milk samples of the PM and AM milkings were obtained from the last four days of each treatment period. The analytical methods of milk samples were the same as those reported previously ${ }^{19}$. Blood samples were taken from jugular and subcutaneous abdominal veins at 4-5 hours after the feeding on the last day of each treatment period. The lipids were extracted from the samples of plasma by the method of Folch et al..$^{5}$, The extracted lipids were fractionated by thin-layer chromatography on plates of silica gel G (E. Merk, A.G., Darmstadt, Germany) using a solvent system of petroleum ether-diethyl ether-acetic acid $(80: 20: 1, v / v)$. The fatty acid compositions of the lipid fractions were determined by the methods reported previously ${ }^{20}$ ). The quantitation of each of the lipid fractions in the plasma was made by the following methods: triglycerides, VAN HANDEL and ZILVERSMIT ${ }^{21)}$; cholesterol and cholesterol esters, ABELL et al. ${ }^{11}$; phospholipids, BartletT ${ }^{41}$; unesterified fatty acids, Patterson ${ }^{13}$. Samples of rumen liquor were taken by stomach tube on the last day of each treatment period and were analysed for volatile fatty acids by the method as described by Matsuoka et al. ${ }^{7}$ 


\section{Results}

Weight percentages of the fatty acids as methyl ester in the alfalfa hay cube, the concentrate mixture, stearic acid and safflower oil are given in Table 2.

In the rumen liquor, the stearic acid treatment had no effect on the molar proportions of volatile fatty acids, and the safflower oil treatment resulted in an increase in the molar proportion of propionic acid and a decrease in that of acetic acid (Table 3 ).

Milk Mean values for the yield and composition of the milk are given in Table 4. Neither the stearic acid nor the safflower oil treatment affected both the yield of milk and the percentage of S.N.F. and of protein in the milk. The percentage of the fat in the milk tended to be increased by the former treatment, but was decreased by the latter treatment.

Mean weight percentages of the fatty acids as methyl ester in the milk fat are given in Table 5. By both treatments, the percentages of the fatty acids from 4:0 to 16:0 in the milk fat decreased, while those of 18:0 and 18:1 in the milk fat were increased.

Blood plasma lipids Concentrations of the various lipid fractions in the plasma from jugular and subcutaneous abdominal veins are given in Table 6. The stearic acid treatment caused an increase in the concentration of triglycerides in the plasma obtained from jugular vein, whereas the safflower oil treatment brought about opposite results. The concentrations of cholesterol esters, free cholesterol and phospholipids in the plasma from jugular and subcutaneous abdominal veins were increased by both treatments. Neither the stearic acid nor the safflower oil treatments safflower oil treatment affected the concentration of unesterified fatty acids in the plasma from jugular and subcutaneous abdominal veins. The concentration of triglycerides was always higher in the plasma from jugular vein than in the plasma from subcutaneous abdominal vein.

The fatty acid compositions of triglycerides, unesterified fatty acids, cholesterol esters and phospholipids in the plasma from jugular vein are given in Table 7. The stearic acid or safflower oil treatment resulted in the increase in the percentage of 18:0 and the decrease of 16:0 in the plasma triglycerides and unesterified fatty acids. The saffower oil treatment resulted in the increase in the percentage of 18:1 in the plasma triglycerides and unesterified fatty acids. The stearic acid treatment had no effect on the fatty acid composition in the plasma cholesterol esters. But the safflower oil treatment resulted in an increase in the percentage of $18: 2$ and a decrease of $18: 3$ in the plasma cholesterol esters. By the stearic acid treatment, the percentages of 18:0 and 18:1 in the plasma phospholipids tended to be increased, while those of $16: 0$ and 18:2 were decreased. By the safflower oil treatment, the the percentage of 18:2 in the plasma phospholipids was increased while those of 16:0, 18:3 and unsaturated fatty acids higher than $18: 3$ tended to be decreased.

\section{Discussion}

All the rations used in the present experiments were readily accepted by the cows and they did not show any signs of digestive or physiological disturbances.

By the safflower oil treatment, the findings in the molar proportions of volatile fatty acids in the rumen liquor agree with the findings of SHAw and ENsoR ${ }^{14)}$ and of others ${ }^{17,227}$.

The results given in Table 6 indicate that the yield of the milk fat was not related to the concentrations of cholesterol esters, unesterified fatty acids or phospholipids in the plasma, 


\section{TANAKA, NakajIma and HAYashI}

Table 2. Compositions of the fatty acids present in the alfalfa hay cube, concentrate mixture, stearic acid and safflower oil

\begin{tabular}{c|cccc}
\hline $\begin{array}{c}\text { Fatty acid* } \\
\text { methyl ester }\end{array}$ & $\begin{array}{c}\text { Alfalfa } \\
\text { hay cube }\end{array}$ & $\begin{array}{c}\text { Concentrate } \\
\text { mixture }\end{array}$ & Stearic acid & Safflower oil \\
\hline $12: 0$ & 1.3 & - & $(\%)^{* * *}$ & - \\
$14: 0$ & 5.2 & 1.0 & - & - \\
$14: 1$ & 1.5 & - & - & - \\
$16: 0$ & 22.7 & 22.0 & 8.6 & 5.4 \\
$16: 1$ & 1.8 & 0.8 & - & - \\
$18: 0$ & 4.6 & 2.1 & 2.4 & 1.6 \\
$18: 1$ & 5.2 & 28.6 & - & 79.5 \\
$18: 2$ & 23.3 & 42.9 & - & 0.3 \\
$18: 3$ & 28.2 & 1.4 & - & - \\
$18: 3<$ & 6.2 & 1.2 & & \\
\hline
\end{tabular}

* Number of carbon atoms : Number of double bonds

** Values expressed as \% of total fatty acid methyl ester

Table 3. The molar proportions of volatile fatty acids in the rumen liquor of the cows in each treatment period

\begin{tabular}{|c|c|c|c|c|c|c|c|c|}
\hline \multirow[b]{2}{*}{ V.F.A. } & \multicolumn{4}{|c|}{ Stearic acid treatment } & \multicolumn{4}{|c|}{ Safflower oil treatment } \\
\hline & $\begin{array}{l}\text { Cows } \\
\text { No. }\end{array}$ & $\begin{array}{l}\text { Preli- } \\
\text { minary } \\
\text { period }\end{array}$ & $\begin{array}{l}\text { Experi- } \\
\text { mental } \\
\text { period }\end{array}$ & $\begin{array}{c}\text { Post-ex- } \\
\text { perimental } \\
\text { period }\end{array}$ & $\begin{array}{l}\text { Cows } \\
\text { No. }\end{array}$ & $\begin{array}{l}\text { Preli- } \\
\text { minary } \\
\text { period }\end{array}$ & $\begin{array}{l}\text { Experi- } \\
\text { mental } \\
\text { period }\end{array}$ & $\begin{array}{l}\text { Post-ex- } \\
\text { perimental } \\
\text { period }\end{array}$ \\
\hline $2: 0$ & $\begin{array}{l}1 \\
2\end{array}$ & $\begin{array}{l}57.6 \\
58.4\end{array}$ & $\begin{array}{l}(\%) \\
57.2 \\
58.2\end{array}$ & $\begin{array}{l}57.0 \\
57.6\end{array}$ & $\begin{array}{l}3 \\
4\end{array}$ & $\begin{array}{l}59.9 \\
59.0\end{array}$ & $\begin{array}{l}(\%) \\
53.1 \\
52.0\end{array}$ & $\begin{array}{l}58.7 \\
59.9\end{array}$ \\
\hline $3: 0$ & $\begin{array}{l}1 \\
2\end{array}$ & $\begin{array}{l}22.0 \\
22.1\end{array}$ & $\begin{array}{l}21.8 \\
22.2\end{array}$ & $\begin{array}{l}22.8 \\
21.6\end{array}$ & $\begin{array}{l}3 \\
4\end{array}$ & $\begin{array}{l}21.3 \\
22.5\end{array}$ & $\begin{array}{l}27.4 \\
27.7\end{array}$ & $\begin{array}{l}23.4 \\
22.4\end{array}$ \\
\hline $4: 0$ & $\begin{array}{l}1 \\
2\end{array}$ & $\begin{array}{l}18.6 \\
18.2\end{array}$ & $\begin{array}{l}19.5 \\
18.2\end{array}$ & $\begin{array}{l}18.8 \\
19.2\end{array}$ & $\begin{array}{l}3 \\
4\end{array}$ & $\begin{array}{l}17.6 \\
17.3\end{array}$ & $\begin{array}{l}17.1 \\
17.3\end{array}$ & $\begin{array}{l}16.6 \\
16.3\end{array}$ \\
\hline $5: 0$ & $\begin{array}{l}1 \\
2\end{array}$ & $\begin{array}{l}1.8 \\
1.3\end{array}$ & $\begin{array}{l}1.5 \\
1.4\end{array}$ & $\begin{array}{l}1.4 \\
1.6\end{array}$ & $\begin{array}{l}3 \\
4\end{array}$ & $\begin{array}{l}1.2 \\
1.2\end{array}$ & $\begin{array}{l}2.4 \\
3.0\end{array}$ & $\begin{array}{l}1.3 \\
1.4\end{array}$ \\
\hline $2: 0 / 3: 0$ & $\frac{1}{2}$ & $\begin{array}{l}2.6 \\
2.6\end{array}$ & $\begin{array}{l}2.6 \\
2.6\end{array}$ & $\begin{array}{l}2.5 \\
2.7\end{array}$ & $\begin{array}{l}3 \\
4\end{array}$ & $\begin{array}{l}2.8 \\
2.6\end{array}$ & $\begin{array}{l}1.9 \\
1.9\end{array}$ & $\begin{array}{l}2.5 \\
2.8\end{array}$ \\
\hline
\end{tabular}

Table 4. Mean daily yield and composition of the milk in each treatment period

\begin{tabular}{|c|c|c|c|c|c|c|c|c|}
\hline \multirow{2}{*}{ ! } & \multicolumn{4}{|c|}{ Stearic acid treatment } & \multicolumn{4}{|c|}{ Safflower oil treatment } \\
\hline & $\begin{array}{c}\text { Cows } \\
\text { No. }\end{array}$ & $\begin{array}{l}\text { Preli- } \\
\text { minary } \\
\text { period }\end{array}$ & $\begin{array}{l}\text { Experi- } \\
\text { mental } \\
\text { period }\end{array}$ & $\begin{array}{c}\text { Post-ex- } \\
\text { perimental } \\
\text { period }\end{array}$ & $\begin{array}{c}\text { Cows } \\
\text { No. }\end{array}$ & $\begin{array}{l}\text { Preli- } \\
\text { minary } \\
\text { period }\end{array}$ & $\begin{array}{l}\text { Experi- } \\
\text { mental } \\
\text { period }\end{array}$ & $\begin{array}{c}\text { Post-ex- } \\
\text { perimental } \\
\text { period }\end{array}$ \\
\hline $\begin{array}{l}\text { Milk yield } \\
\text { (kg/day) }\end{array}$ & $\begin{array}{l}1 \\
2\end{array}$ & $\begin{array}{l}11.9 \pm 0.4^{*} \\
24.6 \pm 0.6\end{array}$ & $\begin{array}{l}11.1 \pm 0.4 \\
24.1 \pm 0.6\end{array}$ & $\begin{array}{l}11.0 \pm 0.4 \\
23.2 \pm 0.8\end{array}$ & $\begin{array}{l}3 \\
4\end{array}$ & $\begin{array}{l}18.0 \pm 0.3 \\
22.8 \pm 0.4\end{array}$ & $\begin{array}{l}17.0 \pm 0.5 \\
22.1 \pm 0.6\end{array}$ & $\begin{array}{l}15.9 \pm 0.6 \\
21.4 \pm 0.5\end{array}$ \\
\hline $\begin{array}{l}\text { Fat } \\
(\%)\end{array}$ & $\begin{array}{l}1 \\
2\end{array}$ & $\begin{array}{l}3.4 \pm 0.1 \\
3.3 \pm 0.1\end{array}$ & $\begin{array}{l}3.8 \pm 0.2 \\
3.5 \pm 0.2\end{array}$ & $\begin{array}{l}3.5 \pm 0.1 \\
3.4 \pm 0.2\end{array}$ & $\begin{array}{l}3 \\
4\end{array}$ & $\begin{array}{l}3.6 \pm 0.2 \\
3.3 \pm 0.1\end{array}$ & $\begin{array}{l}2.9 \pm 0.2 \\
2.5 \pm 0.1\end{array}$ & $\begin{array}{l}3.6 \pm 0.1 \\
3.2 \pm 0.1\end{array}$ \\
\hline$\underset{(\%)}{\text { S. N. F. }}$ & $\begin{array}{l}1 \\
2\end{array}$ & $\begin{array}{l}8.6 \pm 0.2 \\
9.1 \pm 0.1\end{array}$ & $\begin{array}{l}8.6 \pm 0.2 \\
9.0 \pm 0.2\end{array}$ & $\begin{array}{l}8.6 \pm 0.3 \\
9.1 \pm 0.1\end{array}$ & $\begin{array}{l}3 \\
4\end{array}$ & $\begin{array}{l}9.2 \pm 0.2 \\
8.2 \pm 0.1\end{array}$ & $\begin{array}{l}9.1=0.2 \\
8.3=0.2\end{array}$ & $\begin{array}{l}9.2 \pm 0.1 \\
8.2 \pm 0.2\end{array}$ \\
\hline $\begin{array}{l}\text { Protein } \\
(\%)\end{array}$ & $\begin{array}{l}1 \\
2\end{array}$ & $\begin{array}{l}3.5 \pm 0.1 \\
3.5 \pm 0.1\end{array}$ & $\begin{array}{l}3.5 \pm 0.1 \\
3.5 \pm 0.1\end{array}$ & $\begin{array}{l}3.5 \pm 0.1 \\
3.5 \pm 0.1\end{array}$ & $\begin{array}{l}3 \\
4\end{array}$ & $\begin{array}{l}3.6 \pm 0.1 \\
3.0 \pm 0.1\end{array}$ & $\begin{array}{l}3.4 \pm 0.1 \\
2.9 \pm 0.1\end{array}$ & $\begin{array}{l}3.6 \pm 0.1 \\
3.0 \pm 0.1\end{array}$ \\
\hline
\end{tabular}

* Standard deviation 
Dietary Fat and Milk Fat

Table 5. Mean weight percentages of the fatty acids in the milk fat in each treatment period

\begin{tabular}{|c|c|c|c|c|c|c|c|c|}
\hline \multirow{2}{*}{$\begin{array}{c}\text { Fatty acid } \\
\text { methyl } \\
\text { ester }\end{array}$} & \multicolumn{4}{|c|}{ Stearic acid treatment } & \multicolumn{4}{|c|}{ Safflower oil treatment } \\
\hline & $\begin{array}{l}\text { Cows } \\
\text { No. }\end{array}$ & $\begin{array}{l}\text { Preli- } \\
\text { minary } \\
\text { period }\end{array}$ & $\begin{array}{l}\text { Experi: } \\
\text { mental } \\
\text { period }\end{array}$ & $\begin{array}{c}\text { Post-ex- } \\
\text { perimental } \\
\text { period }\end{array}$ & $\begin{array}{c}\text { Cows } \\
\text { No. }\end{array}$ & $\begin{array}{l}\text { Preli- } \\
\text { minary } \\
\text { period }\end{array}$ & $\begin{array}{l}\text { Experi- } \\
\text { mental } \\
\text { period }\end{array}$ & $\begin{array}{c}\text { Post-ex- } \\
\text { perimental } \\
\text { period }\end{array}$ \\
\hline $\begin{array}{l}4: 0+ \\
6: 0\end{array}$ & $\begin{array}{l}1 \\
2\end{array}$ & $\begin{array}{l}2.4 \pm 0.2^{*} \\
2.4 \pm 0.1\end{array}$ & $\begin{array}{c}(\%) \\
2.0 \pm 0.1 \\
1.9 \pm 0.1\end{array}$ & & $\begin{array}{l}3 \\
4\end{array}$ & $\begin{array}{l}2.8 \pm 0.3 \\
2.7 \pm 0.3\end{array}$ & $\begin{array}{c}(\%) \\
1.9 \pm 0.1 \\
1.9 \pm 0.1\end{array}$ & $\begin{array}{l}2.6 \pm 0.2 \\
2.6 \pm 0.1\end{array}$ \\
\hline $8: 0$ & $\begin{array}{l}1 \\
2\end{array}$ & $\begin{array}{l}1.7 \pm 0.2 \\
1.7 \pm 0.1\end{array}$ & $\begin{array}{l}1.3 \pm 0.1 \\
1.3 \pm 0.2\end{array}$ & & $\begin{array}{l}3 \\
4\end{array}$ & $\begin{array}{l}1.7 \pm 0.2 \\
1.7 \pm 0.1\end{array}$ & & $\begin{array}{l}1.7 \pm 0.1 \\
1.6 \pm 0.1\end{array}$ \\
\hline $10: 0$ & $\begin{array}{l}1 \\
2\end{array}$ & $\begin{array}{l}4.7 \pm 0.7 \\
4.4 \pm 0.5\end{array}$ & & & $\begin{array}{l}3 \\
4\end{array}$ & & & \\
\hline $12: 0$ & $\begin{array}{l}1 \\
2\end{array}$ & $\begin{array}{l}5.9 \pm 0.2 \\
5.2 \pm 0.4\end{array}$ & & & $\begin{array}{l}3 \\
4\end{array}$ & & & \\
\hline $14: 0$ & $\begin{array}{l}1 \\
2\end{array}$ & $\begin{array}{l}13.5 \pm 0.4 \\
14.6 \pm 0.5\end{array}$ & $\begin{array}{l}10.1 \pm 0.4 \\
11.5 \pm 0.4\end{array}$ & $\begin{array}{l}13.3 \pm 0.1 \\
14.7 \pm 0.3\end{array}$ & $\begin{array}{l}3 \\
4\end{array}$ & $\begin{array}{l}13.7 \pm 0.1 \\
15.5 \pm 0.6\end{array}$ & $\begin{array}{l}10.5 \pm 0.1 \\
11.0 \pm 0.9\end{array}$ & $\begin{array}{l}13.7 \pm 0.6 \\
15.0 \pm 0.1\end{array}$ \\
\hline $14: 1$ & $\begin{array}{l}1 \\
2\end{array}$ & $\begin{array}{l}1.4 \pm 0.1 \\
1.3 \pm 0.2\end{array}$ & $\begin{array}{l}0.8 \pm 0.1 \\
0.9 \pm 0.1\end{array}$ & & $\begin{array}{l}3 \\
4\end{array}$ & $\begin{array}{l}1.5 \pm 0.1 \\
1.6 \pm 0.1\end{array}$ & & $\begin{array}{l}1.3 \pm 0.1 \\
1.5 \pm 0.1\end{array}$ \\
\hline $16: 0$ & $\begin{array}{l}1 \\
2\end{array}$ & $\begin{array}{l}29.9 \pm 0.5 \\
32.3 \pm 0.6\end{array}$ & $\begin{array}{l}22.2 \pm 0.4 \\
23.0 \pm 0.5\end{array}$ & $\begin{array}{l}29.6 \pm 0.2 \\
33.1 \pm 0.2\end{array}$ & $\begin{array}{l}3 \\
4\end{array}$ & $\begin{array}{l}32.6 \pm 0.6 \\
31.2 \pm 0.5\end{array}$ & $\begin{array}{l}22.8 \pm 0.6 \\
21.6 \pm 0.4\end{array}$ & $\begin{array}{l}31.9 \pm 0.7 \\
32.6 \pm 0.4\end{array}$ \\
\hline $16: 1$ & $\begin{array}{l}1 \\
2\end{array}$ & $\begin{array}{l}1.3 \pm 0.1 \\
1.1 \pm 0.2\end{array}$ & $\begin{array}{l}0.9 \pm 0.1 \\
0.8 \pm 0.1\end{array}$ & $\begin{array}{l}1.3 \pm 0.1 \\
1.0 \pm 0.1\end{array}$ & $\begin{array}{l}3 \\
4\end{array}$ & $\begin{array}{l}1.4 \pm 0.1 \\
1.3 \pm 0.1\end{array}$ & $\begin{array}{l}1.2 \pm 0.1 \\
1.1 \pm 0.1\end{array}$ & $\begin{array}{l}1.4 \pm 0.1 \\
1.4 \pm 0.2\end{array}$ \\
\hline $18: 0$ & $\begin{array}{l}1 \\
2\end{array}$ & $\begin{array}{l}12.3 \pm 0.6 \\
12.0 \pm 0.7\end{array}$ & $\begin{array}{l}23.1 \pm 1.3 \\
21.9 \pm 1.0\end{array}$ & $\begin{array}{l}13.8 \pm 0.6 \\
11.6 \pm 0.2\end{array}$ & $\begin{array}{l}3 \\
4\end{array}$ & $\begin{array}{l}12.1 \pm 0.8 \\
11.6 \pm 0.8\end{array}$ & $\begin{array}{l}18.6 \pm 0.5 \\
18.2 \pm 0.3\end{array}$ & $\begin{array}{l}12.8 \pm 0.6 \\
12.0 \pm 0.4\end{array}$ \\
\hline $18: 1$ & $\begin{array}{l}1 \\
2\end{array}$ & $\begin{array}{l}23.9 \pm 0.7 \\
22.3 \pm 0.5\end{array}$ & $\begin{array}{l}30.5 \pm 0.4 \\
29.1 \pm 0.6\end{array}$ & $\begin{array}{l}24.4 \pm 0.7 \\
21.9 \pm 0.3\end{array}$ & $\begin{array}{l}3 \\
4\end{array}$ & $\begin{array}{l}22.0 \pm 0.4 \\
23.1 \pm 0.8\end{array}$ & $\begin{array}{l}33.2 \pm 0.3 \\
34.9 \pm 1.3\end{array}$ & $\begin{array}{l}22.8 \pm 0.6 \\
23.0 \pm 0.6\end{array}$ \\
\hline $18: 2$ & $\begin{array}{l}1 \\
2\end{array}$ & $\begin{array}{l}2.6 \pm 0.3 \\
2.2 \pm 0.2\end{array}$ & $\begin{array}{l}1.7 \pm 0.1 \\
2.0 \pm 0.1\end{array}$ & $\begin{array}{l}2.1 \pm 0.1 \\
2.0 \pm 0.1\end{array}$ & $\begin{array}{l}3 \\
4\end{array}$ & $\begin{array}{l}1.4 \pm 0.2 \\
1.4 \pm 0.1\end{array}$ & $\begin{array}{l}2.6 \pm 0.2 \\
2.4 \pm 0.1\end{array}$ & $\begin{array}{l}1.7 \pm 0.2 \\
1.3 \pm 0.1\end{array}$ \\
\hline $18: 3$ & $\begin{array}{l}1 \\
2\end{array}$ & $\begin{array}{l}0.6 \pm 0.1 \\
0.6 \pm 0.1\end{array}$ & $\begin{array}{l}0.3 \pm 0.1 \\
0.4 \pm 0.1\end{array}$ & $\begin{array}{l}0.6 \pm 0.1 \\
0.4 \pm 0.1\end{array}$ & $\begin{array}{l}3 \\
4\end{array}$ & $\begin{array}{l}0.7 \pm 0.1 \\
0.5 \pm 0.1\end{array}$ & $\begin{array}{l}0.4 \pm 0.1 \\
0.4 \pm 0.1\end{array}$ & $\begin{array}{l}0.7 \pm 0.1 \\
0.5 \pm 0.1\end{array}$ \\
\hline
\end{tabular}

Table 6. The concentrations of individual lipid fraction of the plasma of the cows in each treatment period

\begin{tabular}{|c|c|c|c|c|c|c|}
\hline & \multicolumn{3}{|c|}{ Stearic acid treatment } & \multicolumn{3}{|c|}{ Safflower oil treatment } \\
\hline & $\begin{array}{l}\text { Preli- } \\
\text { minary } \\
\text { period }\end{array}$ & $\begin{array}{l}\text { Experi- } \\
\text { mental } \\
\text { period }\end{array}$ & $\begin{array}{c}\text { Post-ex- } \\
\text { perimental } \\
\text { period }\end{array}$ & $\begin{array}{l}\text { Preli- } \\
\text { minary } \\
\text { period }\end{array}$ & $\begin{array}{l}\text { Experi- } \\
\text { mental } \\
\text { period }\end{array}$ & $\begin{array}{c}\text { Post-ex- } \\
\text { perimental } \\
\text { period }\end{array}$ \\
\hline Triglycerides & \multicolumn{3}{|c|}{$(\mathrm{mg} / 100 \mathrm{ml})$} & \multicolumn{3}{|c|}{$(\mathrm{mg} / 100 \mathrm{~m} l)$} \\
\hline $\begin{array}{l}\text { J. V.* } \\
\text { S. V.** }\end{array}$ & $\begin{array}{r}15.3 \\
6.2\end{array}$ & $\begin{array}{r}21.7 \\
8.3\end{array}$ & $\begin{array}{r}16.2 \\
6.0\end{array}$ & $\begin{array}{r}18.4 \\
7.4\end{array}$ & $\begin{array}{r}10.0 \\
6.8\end{array}$ & $\begin{array}{r}18.1 \\
7.6\end{array}$ \\
\hline $\begin{array}{l}\text { Cholesterol esters } \\
\text { J. V. } \\
\text { S. V. }\end{array}$ & $\begin{array}{l}180.0 \\
165.4\end{array}$ & $\begin{array}{l}244.7 \\
207.3\end{array}$ & $\begin{array}{l}193.5 \\
181.8\end{array}$ & $\begin{array}{l}203.4 \\
197.2\end{array}$ & $\begin{array}{l}270.5 \\
259.2\end{array}$ & $\begin{array}{l}204.7 \\
203.0\end{array}$ \\
\hline $\begin{array}{l}\text { Free cholesterol } \\
\text { J. V. } \\
\text { S. V. }\end{array}$ & $\begin{array}{l}45.0 \\
43.3\end{array}$ & $\begin{array}{l}59.0 \\
51.0\end{array}$ & $\begin{array}{l}46.1 \\
43.1\end{array}$ & $\begin{array}{l}55.4 \\
51.7\end{array}$ & $\begin{array}{l}66.4 \\
66.2\end{array}$ & $\begin{array}{l}51.0 \\
50.8\end{array}$ \\
\hline $\begin{array}{l}\text { Phospholipids } \\
\text { J. V. } \\
\text { S. V. }\end{array}$ & $\begin{array}{l}212.0 \\
201.9\end{array}$ & $\begin{array}{l}269.1 \\
249.8\end{array}$ & $\begin{array}{l}241.9 \\
225.0\end{array}$ & $\begin{array}{l}244.7 \\
231.2\end{array}$ & $\begin{array}{l}332.8 \\
317.2\end{array}$ & $\begin{array}{l}246.7 \\
230.5\end{array}$ \\
\hline $\begin{array}{l}\text { Unesterified fatty acids } \\
\text { J. V. } \\
\text { S. V. }\end{array}$ & $\begin{array}{l}8.3 \\
8.7\end{array}$ & $\begin{array}{r}10.2 \\
8.2\end{array}$ & $\begin{array}{r}10.1 \\
9.8\end{array}$ & $\begin{array}{l}7.8 \\
7.5\end{array}$ & $\begin{array}{r}10.1 \\
9.8\end{array}$ & $\begin{array}{l}8.4 \\
7.6\end{array}$ \\
\hline
\end{tabular}

* Jugular vein, ** Subcutaneous abdominal vein

The figures are mean values of two cows. 
TANAKa, NaKaJima and Hayashi

Table 7. Weight percentages of the fatty acids of individual lipid fraction in the plasma from jugular vein of the cows in each treatment period

\begin{tabular}{|c|c|c|c|c|c|c|}
\hline \multirow[b]{2}{*}{$\begin{array}{c}\text { Fatty acid methyl } \\
\text { ester }\end{array}$} & \multicolumn{3}{|c|}{ Stearic acid treatment } & \multicolumn{3}{|c|}{ Safflower oil treatment } \\
\hline & $\begin{array}{c}\text { Preli- } \\
\text { minary } \\
\text { period }\end{array}$ & $\begin{array}{l}\text { Experi- } \\
\text { mental } \\
\text { period }\end{array}$ & $\begin{array}{c}\text { Post-ex- } \\
\text { perimental } \\
\text { period }\end{array}$ & $\begin{array}{l}\text { Preli- } \\
\text { minary } \\
\text { Period }\end{array}$ & $\begin{array}{l}\text { Experi- } \\
\text { mental } \\
\text { period }\end{array}$ & $\begin{array}{c}\text { Post-ex- } \\
\text { perimental } \\
\text { period }\end{array}$ \\
\hline \multicolumn{7}{|l|}{ Triglycerides } \\
\hline $16: 0$ & 33.9 & 28.5 & 33.7 & 33.1 & 21.0 & 35.2 \\
\hline $16: 1$ & 3.7 & 4.0 & 4.4 & 3.2 & 2.2 & 3.0 \\
\hline $18: 0$ & 33.8 & 43.6 & 33.9 & 36.6 & 44.5 & 34.5 \\
\hline $18: 1$ & 18.5 & 16.5 & 19.4 & 18.1 & 23.7 & 18.4 \\
\hline $18: 2$ & 10.0 & 7.3 & 8.5 & 8.9 & 8.5 & 8.8 \\
\hline \multicolumn{7}{|l|}{ Unesterified fatty acids } \\
\hline $16: 0$ & 45.2 & 38.7 & 45.6 & 40.4 & 31.0 & 39.9 \\
\hline $16: 1$ & 3.8 & 3.9 & 3.5 & 2.5 & 2.7 & 3.2 \\
\hline $18: 0$ & 23.9 & 36.1 & 23.7 & 22.8 & 31.0 & 25.0 \\
\hline $18: 1$ & 18.7 & 16.0 & 19.6 & 28.3 & 32.0 & 26.9 \\
\hline $18: 2$ & 8.3 & 5.1 & 7.5 & 5.9 & 3.1 & 4.9 \\
\hline \multicolumn{7}{|l|}{ Cholesterol esters } \\
\hline $16: 0$ & 5.4 & 4.1 & 5.2 & 5.4 & 4.1 & 5.9 \\
\hline $16: 1$ & 3.5 & 3.1 & 3.5 & 3.3 & 2.4 & 3.4 \\
\hline $18: 0$ & 1.7 & 1.0 & 1.6 & 1.4 & 1.2 & 1.3 \\
\hline $18: 1$ & 4.0 & 4.3 & 4.2 & 5.7 & 4.5 & 5.6 \\
\hline $18: 2$ & 71.0 & 71.8 & 71.6 & 66.6 & 75.5 & 66.7 \\
\hline $18: 3$ & 10.4 & 11.4 & 9.6 & 12.8 & 8.7 & 12.1 \\
\hline $20: 3$ & 0.8 & 0.8 & 0.7 & 0.5 & 0.4 & 0.4 \\
\hline $20: 4$ & 3.1 & 3.3 & 3.5 & 4.2 & 3.1 & 4.4 \\
\hline \multicolumn{7}{|l|}{ Phospholipids } \\
\hline $16: 0$ & 18.5 & 16.0 & 19.2 & 17.6 & 15.0 & 18.2 \\
\hline $16: 1$ & 0.5 & 0.5 & 0.6 & 0.7 & 0.5 & 0.6 \\
\hline $18: 0$ & 25.6 & 29.3 & 26.7 & 29.0 & 28.8 & 29.4 \\
\hline $18: 1$ & 8.9 & 12.4 & 9.7 & 11.5 & 13.1 & 12.3 \\
\hline $18: 2$ & 32.7 & 29.0 & 31.1 & 26.0 & 32.6 & 26.1 \\
\hline $18: 3$ & 2.2 & 2.5 & 1.7 & 2.5 & 1.3 & 2.1 \\
\hline $20: 3$ & 5.3 & 4.9 & 5.2 & 4.8 & 3.7 & 4.5 \\
\hline $20: 4$ & 4.0 & 3.7 & 4. 1 & 4.5 & 3.0 & 4.1 \\
\hline $22: 5$ & 1.0 & 0.7 & 0.8 & 1.8 & 1.0 & 1.3 \\
\hline $22: 6$ & 0.9 & 0.7 & 0.6 & 1.4 & 0.8 & 1.1 \\
\hline
\end{tabular}

The figures are mean values of two cows.

but a decrease in the concentration of the plasma triglycerides resulted in a decrease in the yield of the milk fat. This findings coinclde with the findings of Barry et al. ${ }^{3}$ and ANNIsoN et $a l .^{2}$, who reported that a portion of the milk fat of cows was derived from the plasma triglycerides which circulated as chylomicrons and low-density lipoproteins. On the other hand, by supplementing the diet with palmitic or stearic acid, MOORE et al. ${ }^{10}$ investigated the relationship between the composition of the blood plasma lipids and the yield of the milk fat 


\section{Dietary Fat and Milk Fat}

of cows, and they reported a positive curvilinear relationship between the yield of the milk fat and the concentration of the plasma unesterified fatty acids. In their experiment, blood samples were taken from subcutaneous abdominal vein, and the high concentration in the venous blood draining from the mammary gland would mean unutilization of the fatty acids in the gland. In the present experiments, the effect of dietary fat on the concentration of the plasma triglycerides was not so obvious in the plasma from subcutaneous abdominal vein as in the plasma from jugular vein.

The present experiments did not show any appreciable increase in the percentage of $18: 2$ in the milk fat, in spite of an increased dietary intake of 18:2. Similar effects on the fatty acid composition of the milk fat of the cows given diet supplemented with vegetable oils rich in the $\mathrm{C}_{18}$ dienoic and trienoic fatty acids have been reported by STEELE and MoORE ${ }^{\left.16,1^{6}\right)}$, PERRY and MACLeOD ${ }^{12)}$, Steele et $a l .^{18}$ and TANAKA ${ }^{19}$. This result can be explained as a result from the hydrogenation of $18: 2$, taken place in the rumen prior to absorption from the digestive tract. The increase of the percentage of $18: 1$ in the milk fat by the safflower oil treatment may have arisen in the two different possible processes: the first process is that the 18:2 contained in safflower oil is completely hydrogenated by rumen micro-organisms and the resulting 18:0 is incorporated into the blood plasma triglycerides and 18:0 is taken up by the mammary gland where $18: 1$ is synthesized by the desaturation of $18: 0^{2,6}$. The second is that the hydrogenation of a part of 18:2 in the rumen may be imcomplete and some of the resulting 18:1 is absorbed into the blood stream and incorporated directly into milk triglycerides in the mammary gland. Presumably, desaturation of 18:0 in the mammary gland may play a greater part in increasing the percentage of 18:1 in the milk fat of the cows given supplementary stearic acid, since, in the plasma triglycerides, the percentage of 18:0 was increased and that of 18:1 was unaltered or even slightly decreased.

It seems difficult to put forward a precise explanation for the findings that the percentages and the yields of the fatty acids from 4:0 to 16:0 in the milk fat were decreased by both treatments. The amount of the milk fat production in the cow is governed largely by the activities of two metabolic processes. In the first of these, acetate and $\beta$-hydroxybutyrate are taken from the blood and utilized as precursors in the de novo synthesis in the mammary gland of the fatty acids from 4:0 to $16: 0$, which are then incorporated into milk triglycerides ${ }^{2,6}$. In the second process, triglycerides (containing mainly 16:0, 18:0 and 18:1) circulating in the blood stream as chylomicrons and low-density lipoproteins $(d<1.019)$ are taken up by the mammary gland ${ }^{2,31}$. Therefore, the reduction in the supply to the mammary gland of acetate or $\beta$-hydroxybutyrate would be expected to decrease the output of the short and medium-chain fatty acids in the milk fat and this would have occurred by the safflower oil treatment which induced a decrease in the molar proportion of acetic acid in the rumen liquor. In the present experiments, however, the stearic acid treament did not alter the molar proportion of acetic acid in the rumen liquor. This may be explained by the findings of MOORE and STEELE ${ }^{9)}$ who suggested that the increased uptake by the mammary gland of long-chain fatty acids $\left(\mathrm{C}_{18}\right.$-fatty acids) from the plasma triglycerides inhibits the de novo synthesis in the mammary gland of short-chain fatty acids from acetate or $\beta$-hydroxybutyrate, since the increased concentrations of long-chain fatty acids in the mammary gland inhibit acetyl-CoA carboxylase, which catalyses the rate limiting step in fatty acid synthesis in the bovine mammary gland.

By the safflower oil treatment, the increased amount of 18:2 in the plasma was found mainly in the cholesterol esters and phospholipids. This finding is in agreement with the 


\section{TANAKA, NAKAJIMA and HAYASH}

results of NOBLE et al.11) and MOORE el al. ${ }^{81}$, who showed that when sheep were given intraruminal or intra-abomasal infusions of maize oil, the absorbed 18:2 was selectively incorporated into the plasma phospholipids and cholesterol esters and only to a very limited extent into the plasma triglycerides and unesterified fatty acids. Although it is generally accepted that the low percentage of 18:2 in the milk fat of cows is derived from an extensive hydrogenation of dietary 18:2, which is taken place in the rumen, the results of the present study favour a view that the 18:2 escaped from the hydrogenation is selectively incorporated into those lipid fractions of the blood which can not donate fatty acids to the mammary gland for milk fat synthesis.

\section{References}

1) Abell, L.L., B.B. Levy, B.B. Brodie, and F.E. Kendall, J biol Chem 195: 357-366. 1952.

2) Annison, E.F., J.L. Linzell, S. Fazakertey, and B.W. Nichols, Biochem J 102: 637-647. 1967.

3) Barry, J.M., W. Bartley, J.L. Linzell, and D.S. Robinson, Biochem J 86: 6-11. 1963.

4) Bartlett, G.R., J biol Chem 234: 466-467. 1959.

5) Folch, J., M. Lees, and G.H. Sloan-StanLeY, J biol Chem 226: 497-509. 1957.

6) Linzell, J.L., E.F. Annison, S. Fazakerley, and R.A. Leng, Biochem J 104: 34-41. 1967.

7) Matsuoka, S., E. Ueyama, and Y. Hirose, Jap J zootech Sci 40: 160-165. 1969.

8) Moore, J.H., R.C. Noble, and W. Steele, Br J Nutr 23: 141-152. 1969.

9) Moore, J.H., and W. Steele, Proc Nutr Soc 27: 66-70. 1968.

10) Moore, J.H., W. Steele, and R.C. Noble, J Dairy Res 36: 383-392. 1969.

11) Noble, R.C., W. Steele, and J.H. Moore, Br J Nutr 23: 709-714. 1969.

12) Perry, F.G., and G.K. MacLeod, J Dairy Sci 51: 1233-1238. 1968.

13) Patterson, D.S.P., Res vet Sci 4: 230-237. 1963.

14) Shaw, J.C., and W.L. ENSOR J Dairy Sci 42: 1238-1240. 1969.

15) Steele, W., and J.H. Moore, J Dairy Res 35: 223-235. 1968.

16) Steele, W., and J.H. Moore, J Dairy Res 35: 343-352. 1968.

17) Steele, W., R.C. Nobae, and J.H. Moore, J Dairy Res 38: 43-48. 1971.

18) Steele, W., R.C. Noble, and J.H. Moore, J Dairy Res 38: 49-56. 1971.

19) TanaKa, K, Jap J zootech Sci 41: 254-261. 1970.

20) Tanaka, K., and H. Hayaashi, Jap J zootech Sci 42: 582-592. 1971.

21) Van Handel, E., and D.B. Zilversmit, J Lab clin Med 50: 152-157. 1957.

22) Varman, P.N., L.H. Schultz, and R.E. Nichols, J Dairy Sci 51: 1956-1963. 1968. 


\title{
ステアリン酸およびサフラワー油給与が乳牛の血漿中脂質
} および乳脂肪におよぼす影響について

\author{
田中桂一・中島岩男・林英夫
}

岐阜大学農学部，各務原市 504

泌乳中の乳牛に脂肪突給与したとき，その脂肪の性質 (三に脂肪酸組成)によって乳脂肪分泌儿およぼ寸影㭛は 著しく異なることが知られている。本実験は性質の異な る2つの脂肪，寸なわちステフリン酸しサフラワー油を それぞれ乳牛に給与したとき，血漿中脂質和よび乳脂肪 にどのような影籍をおよぼすか検討するためにおこなっ t.

论乳中のホルスタイン種乳牛 4 頭索供試し， 2 頭をス テアリン酸給与群さし，他の 2 頭をサフラワ一油給与群 とした. 実験は (1) 前対照期, (2) 脂肪給与期, (3) 後対照期の 3 期間とし，1処理期間をそれぞれ21日と した. 脂肪給与量は 1 日 1 頍当引 $400 \mathrm{~g}$ とした。

第一青内溶液中の揮発性脂肪酸のモル比はサフラワー 油給与によって酷酸注減少し,プロピオン酸は增加し た.

乳脂率はステフリン酸給与によって増加の傾向を示 し, サフラワー油給与によって著しく減少した。

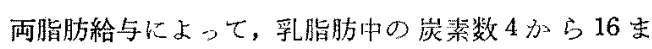
での脂肪酸法減少し, 18:0 18:1 は增加した。

頙静脈血中のトリグリセリドの䟴安はステフリン酸給 与によって增加し，サフラロー油給与によって減少し た. 雨脂肪給与によって, 頸静脈血中および腹皮下静脈 血中のコレステロールェステル，遊離型コレステロール おるよびリン脂質の浱度は增加した。

血等中のトリグリセリドおよび游離型脂肪酸分画て は，両脂游給与によって，18:0 は增加し，16:0 は隇少 した、さらにサフラワー油层給与したとき，18:1 璔加 した．血墏中のコレステロールエステル分画では，サフ ラワー油給与によって，18:2 は增加し，18:3 注減少し た，血漿中のリン脂質分画において，ステアリン酸給与 によって，18:0 と 18:1 は増加の傾向にあり，16:0 と 18:2 は減少の傾向にあった.一方サフラワー油給与に よって，18:2 は堌加した。 